\title{
A Proposed Framework Based on Literature Review of Online Contextual Mental Health Services to Enhance Wellbeing and Address Psychopathology During COVID-19
}

\author{
Ahmet Tanhan ${ }^{1,2 *}$, K. Fatih Yavuz ${ }^{3,4}$, J. Scott Young ${ }^{5}$, Ahmet Nalbant ${ }^{6}$, Gökmen Arslan ${ }^{7}$, Murat Yıldırım ${ }^{8,9}$, \\ Sevinç Ulusoy ${ }^{10}$, Emel Genç ${ }^{11}$, Erol Uğur ${ }^{12}$, Illhan Çiçek ${ }^{13}$
}

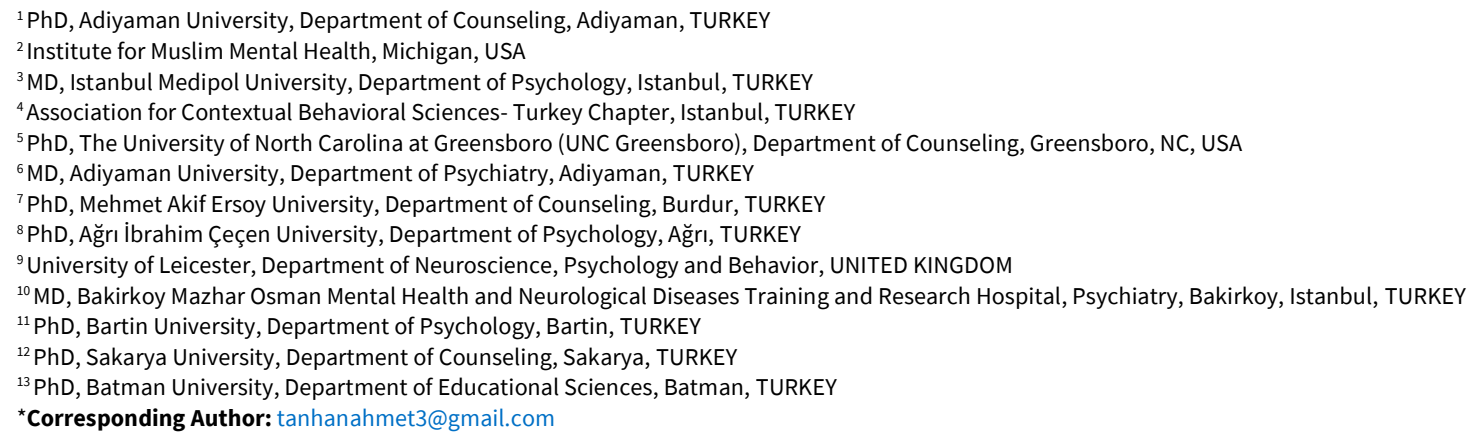

Citation: Tanhan A, Yavuz KF, Young JS, Nalbant A, Arslan G, Yıldırım M, Ulusoy S, Genç E, Uğur E, Çiçek I. A Proposed Framework Based on Literature Review of Online Contextual Mental Health Services to Enhance Wellbeing and Address Psychopathology During COVID-19. Electron J Gen Med. 2020;17(6):em254. https://doi.org/10.29333/ejgm/8316

ARTICLE INFO

Received: 10 May 2020

Accepted: 25 May 2020

\begin{abstract}
Coronavirus disease (COVID-19) became a pandemic and is causing unprecedented biopsychosocial, spiritual and economic issues across the world while mostly affecting unprivileged populations. Turkey has gradually implemented new regulations, which slowly affected the entire country and increased the need for mental health services disproportionally. We conducted a comprehensive literature review on mental health in Turkey during COVID-19. There was no well-grounded peer-reviewed manuscripts or projects utilized a framework. Therefore, we wrote this manuscript to provide a conceptual framework grounded in ecological systems theory, acceptance and commitment therapy, and community-based participatory action research to introduce contextually evidence-based online mental health services: hotline, psychiatric interview, counseling, and Read-Reflect-Share group bibliotherapy. The framework aims to (1) address biopsychosocial spiritual and economic issues, (2) enhance wellbeing, and (3) empower the mental health profession in research and practice. Our preliminary findings and clinical experience indicated that the proposed framework and interventions derived from the framework enhanced wellbeing and decreased psychopathological symptoms in experimental group compared to control groups. Based on the preliminary analysis, most of the online, phone based, or face-to-face mental health services introduced in this manuscript were highly recommended by the participants to be provided to general public during and after COVID-19. Mental health professionals and authorities can use the proposed framework and interventions to develop interventions and research in order to alleviate pandemic-based biopsychosocial spiritual and economic issues and enhance wellbeing.
\end{abstract}

Keywords: COVID-19, online mental health, acceptance and commitment therapy, psychopathology, positive psychology, ecological system theory, community based participatory action research, social justice, pandemic, telemental health

\section{INTRODUCTION}

The coronavirus disease 2019 (COVID-19), previously known as 2019 novel coronavirus, is caused by the virus named severe acute respiratory syndrome coronavirus 2 (SARS-CoV-2) (1). COVID-19 has been identified as originating in Wuhan, Hubei province, China (2). The disease's most well-known symptoms are fever, cough, and shortness of breath. The virus widely and rapidly spread worldwide and became a pandemic causing unprecedented biopsychosocial, spiritual and economic issues. However, it seems that the results affect underdeveloped and developing countries and specifically unprivileged communities more severely across a range of biopsychosocial spiritual and economic perspectives, causing long-term social injustice. One of the most important impacts in the difficult COVID-19 process is mental health due to the fact that physical health is partially based on mental and emotional stability. Furthermore, mental health issues including depression, stress, anxiety (3-8), fear (9-11), physical isolation 
and feeling alone (12), behavioral issues $(4,13)$, possible posttraumatic stress disorders (3), and burnout (14) can affect large numbers of individuals if these issues are not well addressed. Such a range of mental health issues creates stress at individual, group, community, and public levels which affects all echelons of society. These issues are exacerbated by myths and lack of information about COVID-19 (3) and lead people to utilize unscientific and unprofessional ways to address the issues, which lead the first suicide for a Bangladeshi due to suspecting himself having COVID-19 and others' negative attitudes toward him (15). During COVID-19 there are many grounded research (16) in biomedical area yet not in mental health. Therefore, mental health topics require more contextual and evidence-based research, which lacks in literature, to alleviate pain and suffering and enhance wellbeing. Researchers have called for such mental health research and policy regulations to serve all individuals more effectively (7,17-20).

\section{COVID-19 and its Biopsychosocial Spiritual and Economic Results in Turkey}

Turkey, as an upper-middle income country, has relatively struggled to address COVID-19 and related results since January $10^{\text {th }}, 2020$ when the Ministry of Health in Turkey set up the coronavirus scientific advisory board with 26 medical experts (later increased to 31). However, none of these experts were from mental health areas and these experts did not primarily emphasize mental health care of individuals. In Turkey, the ministry of health diagnosed the first COVID-19 case on March $11^{\text {th }}$ and as of May $16^{\text {th }}$, the confirmed total statistics related to COVID-19 include 148,067 diagnosed cases, 4,096 deaths, 108,137 recoveries, and 1,589,625 tests (21). On March $11^{\text {th }}, 2020$ a new process was initiated that gradually, yet dramatically, affected nearly all aspects of daily life, including health, education, religion-spiritual, social, transportation, industry, and economy. More specifically, the central government authorities recommended to maintain physical and social distance rules, online or distant education, vacating all schools and related buildings, staying at home, and stopping inner and across the country transportations. They also gradually closed places of worship, held a home confinement for some ages till a second order countrywide, created a lockdown to get in or out of almost all 81 provinces and a special lockdown for 31 metropolitan provinces for the weekends' requiring home confinement, banning opening most work places and social gathering environments. The ministry of health constantly informed people about COVID-19 and the number of diagnosed cases, deaths, and recovery each day. All these were gradual over a two-week period and yet not expected by many people and therefore brought physical and social isolation that seem to contribute to the development of biopsychosocial spiritual and economic issues ranging from mild to severe levels. Physical and social isolation have the potential to have serious mental and physical impact that authorities in some countries, such as Sweden, did not want to deal with and therefore did not take any precautions like lockdowns.

The number of clients applying for mental health services at a state university counseling clinic where the first author leads mental health services and research increased disproportionally during this difficult COVID-19 process. The mental health profession is under structured in Turkey and is largely organized from a medical perspective meaning there is little emphasis on mental health and specifically counseling or therapy. Moreover, the focus is typically on psychiatry making the psychiatric services overloaded and mostly resulting in interviews with five to ten minutes with each patient. As a result, most of the times visiting a public psychiatrist is reserved for individuals who have a serious mental illness and who need psychotropic medication.

The biopsychosocial, spiritual and economic impacts have steadily increased. This has led to the ministry of health adding some social science experts in mental health, spirituality or religiosity, sociology, and statistic under the name of social sciences board on April $7^{\text {th }} 2020$ to the already constructed coronavirus scientific advisory board. The government, then called for experts to improve social services including mental health supports, having recognized the need for widespread services. Many first line health providers, especially those in Istanbul, were overwhelmed with clients. For example, employers were unable to open their workplaces, employees were unable to make money, slight economic recession (Turkish Lira losing its value against gold and other currencies), mental health providers faced demands and yet did not have appropriately well-structured systems (e.g., online or phone based) to help with the issues because most of them were required to stay at home and work in shifts so that workplaces are not overcrowded. Millions of students were suddenly away from their schools and were struggling to follow distance education requirements from a newly established education channel and about eight million college students leaving their colleges, dormitories, friends, and starting to live with their families while striving to find a way to follow their courses online. Education in Turkey stopped for one to two weeks based on institutions and then resumed yet many individuals struggled with this new phase which required being able to afford technology including internet, and access to a computer or a smart phone. Many individuals and especially college students and clients at the counseling clinic with low socioeconomic status (SES) reached out to the first author asking how they can resume their courses and sessions in this low SES context, which exacerbated their mental health issues. Researchers found that college students in general have higher level of depression (22).

Human beings have biopsychosocial and spiritual needs and therefore need socialization to learn and contribute to society and have a meaningful life (23). However, in COVID-19 process, many individuals face home confinement and physical and social distancing that might lead to technology and internet addiction, which could further exacerbate mental health issues. Çiçek et al. (18) conducted a study in Turkey during COVID-19 and found $72 \%$ of the participants reported they spent more time each day on social media and the internet compared to the pre COVID-19 pandemic period. Of these $\% 1$ reported spending thirteen more hours, $5 \%$ reporting twelve more hours, $28 \%$ reported four to seven more hours, and $66 \%$ reported one to three more daily hours on the internet. Additionally, $46 \%$ percent of these participants reported that they do not follow professional news on media or online. The researchers found that participants had mild levels of depression and anxiety and a moderate level of psychological inflexibility. Additional time spent on the social media compared to pre-COVID-19 epidemic was moderately correlated with depression, anxiety, and psychological inflexibility, whereas internet use had low to moderate correlation with all three scales. In the same study, psychological inflexibility significantly predicted depression 
and anxiety, suggesting the possible effect of interventions if they are designed from an Acceptance and Commitment Therapy (ACT) perspective.

It is crucial to acknowledge the need and advantage of online mental health while being cautious to pay attention to possible disadvantages $(8,19,20,24)$. Similarly, how people and mental health providers utilize internet, media, and social media in such difficult times to empower society is also worth attention $(3,25)$. Therefore, technology and internet use should be considered mindfully and contextually when addressing mental health issues $(18,24,26)$.

\section{Online Mental Health Services in Turkey}

It is apparent that there is a need to provide contextually relevant and evidence based mental health services to address the issues, alleviate suffering, and enhance wellbeing. Physical and social isolation are one of the most harmful ways to deteriorate the human immune system, which could exacerbate already existing illnesses and cause some new biopsychosocial spiritual and economic issues (e.g., domestic violence, technology addiction). This could turn into psychopathology especially if the person is isolated and their issues are not professionally addressed. For example, many individuals with majority being college students reached out to the first author for counseling services or Online Read-ReflectShare (ORRS) bibliotherapy groups at the clinic where the author lead mental health services and research. Majority of these individuals reported loneliness, stress, anxiety, and academic and interpersonal issues. Some of them also reported that they had these issues pre COVID-19 yet they got more intense during the pandemic.

Mental health professionals should take responsibility both to address psychopathology and cultivate wellbeing. Therefore, in such difficult COVID-19 times, it is also possible to cultivate meaningful inner and interrelationship value alignment if people are able to regularly utilize direct professional mental health services or follow the resources and activities grounded on a well-grounded contextually appropriate mental health perspective. Therefore, the physical and social isolation, overuse of technology and internet, and ongoing biopsychosocial spiritual and financial issues should be considered contextually to construct effective services. The lack of sufficient mental health resources put the Chinese people with COVID-19 and their relatives in a difficult situation when they were unable to benefit from resources (27). Similarly, in Turkey, people need more organized and systemic mental health services. To our knowledge, there are no formal public online or telephone based mental health services except for one-time phone-based hotlines, which are designed to intervene and take the person to the hospital. Providing regular counseling or therapy through public institutions can take months between sessions, which is not effective considering the nature of mental health issues and especially during the COVID-19 outbreak. It is important to note that some individuals and mental health companies or associations in Turkey strive to provide online mental health services and to conduct research. However, most of these efforts are not contextually appropriate, well-organized, comprehensive, and evidence-based. Researchers in some other countries found telemental health or online counseling services to be very effective (28-33). Some of the authors of this current study provided limited online or phone based mental health services (individual counseling, psychiatric services, or Online Read-
Reflect-Share [ORRS] group bibliotherapy) in Turkey during COVID-19 and found the services to be very effective.

\section{Purpose of the Present Study}

In this manuscript, we provided a conceptual framework grounded in ecological systems theory, community-based participatory action research, and ACT to introduce a set of contextually situated and evidence-based online mental health services: hotline; psychiatric interviews, counseling, and ORRS group bibliotherapy. We aimed to (1) address biopsychosocial, spiritual, and economic issues, (2) enhance wellbeing, and (3) strengthen mental health profession in the long run in research and practice. Following the first COVID-19 case in Turkey, the counseling clinic where the first author leads mental health services and research, adopted some of the proposed interventions and moved all services online, serving 450 clients, with 240 being supported through ORRS bibliotherapy groups. During the COVID-19 process, the clinic received very heavy demand for participation in ORRS group bibliotherapy. The participants' satisfaction with each of the eight session ranged from $90 \%$ to $97 \%$, and $95 \%$ of the participants recommending ORRS groups to others during COVID-19. Researchers found face-to-face eight session ReadReflect-Share (RRS) group bibliotherapy enhancing wellbeing and alleviating depression and anxiety symptoms compared to a control group (34). Based on all these factors, and especially the central government belated calls for mental health projects, on March $31^{\text {st }} 2020$, we first got in touch with the authorities to stress the need for mental health services to address biopsychosocial spiritual and economic issues and enhance wellbeing. We completed our project on fifth of April and shared it with the authorities. Then, we wrote this paper partially utilizing our submitted project entitled "Addressing biopsychosocial, spiritual and economic issues and enhancing wellbeing through online mental health services within COVID19 context" to improve already existing mental health services and develop new services in Turkey with the proposed framework for contextually and evidence- based interventions.

\section{METHOD}

A set of common key identifiers were searched on research databases including, Google Scholar, PubMed, ERIC, EBSCO, SCOPUS, Education Full Text, Web of Science, and Psyclnfo to conduct a thorough literature review specifically focusing on online or phone based mental health in Turkey during COVID19. The search terms were comprised of a combination of "Turkey, mental health, online mental health, phone based mental health" and one of the following words: COVID-19, coronavirus. Inclusion criteria were to be written in Turkish or English and published as a peer-reviewed manuscript in 2020, address mental health as the primary goal of the manuscript, and elaborate on mental health rather than just mentioning it with few words. In this manuscript, we have followed the steps mentioned above and applied the systematical literature review method.

\section{RESULTS}

A few articles were identified that addressed COVID-19 within Turkey yet nearly all focused solely on biomedical issues, which is not surprising considering the authorities 
Table 1. Foundations of Acceptance and Commitment Therapy

\begin{tabular}{lll}
\hline Functional Contextualism & Relational Frame Theory & Psychological Flexibility and Inflexibility Models \\
\hline $\begin{array}{l}\text { A pragmatic philosophical worldview; } \\
\text { stresses focusing and utilizing what is }\end{array}$ & Focuses on the role of language and cognition & Flexibility Model: explains wellness, liveness, positive \\
workable, functional, useful in life & & psychology, flexibility \\
& & $\begin{array}{l}\text { Inflexibility Model: explains suffering, pathology, } \\
\text { rigidity, inflexibility }\end{array}$ \\
\hline
\end{tabular}

Note. We borrowed the table from Tanhan (2019) with permission.

constant call for and providing budget for such research during the pandemic. Based on our comprehensive review, the researchers focused on the history of epidemics (35), anesthesiology specialists and residents' attitudes toward people with the virus (36), paying attention to intensive care units (37), the nature (38) and the progress of the disease (39). Some research focused on other social aspects including the effects of COVID-19 on the economy (40) and the general public (41) to mention a few.

An exception, Çiçek and his colleagues (18) who surveyed 514 teachers or school principals regarding their levels of depression, anxiety, and psychological inflexibility during COVID-19 process. They found mild levels of depression and anxiety and a moderate level of psychological inflexibility among respondents, as we have provided more information on the study above. Psychological inflexibility, an important ACT terminology, explained a significant amount of depression and anxiety. The researchers recommended developing mental health services from an ACT perspective. Unfortunately, none of these researchers provided well-grounded evidence-based biopsychosocial, spiritual face-to-face, online, or phone based interventions for the COVID-19 context nor did they provide a well-established framework for their study.

All in all, the researchers found that COVID-19 is causing many biopsychosocial, spiritual and economic issues for almost all of humanity including Turkey. Mental health professionals, through research and service provision, show a great effort worldwide to understand and address these issues and enhance wellbeing. We found similar results in Turkey and yet less organized mental health research and services to address the issues and enhance wellbeing. There is a great lack of contextually and evidence-based appropriate mental health services and research. Therefore, we have responded to the belated project calls by submitting our project to the central government. Following the first COVID-19 case in the country, the counseling clinic adopted the proposed conceptual framework and interventions and switched all the individual, group and other mental health services to online serving 450 clients, with 240 being in online RRS groups. The participants' satisfaction with each of the eight online RRS sessions ranged from $90 \%$ to $97 \%$, and $\% 95$ of the participants recommended providing face-to-face or online RRS to others during COVID-19. Based on our results from the clinic which we have utilized the framework for the last three years, the proposed framework and mental health interventions seem to be effective to address the issues and enhance wellbeing in this difficult COVID-19 experience.

\section{Conceptual Framework}

Tanhan and Young (42) explained the need for researchers constructing or utilizing available concept maps, conceptual frameworks, and/or theoretical frameworks for systematic robust mental health research and services. Though parts of the following definition could be used interchangeably, a concept map is a visual figure of main concepts for a study; a conceptual framework is the primary concepts and theories and the interaction among them in the larger perspective of a line of research. Finally, a theoretical framework is the dynamics among concrete constructs that can be empirically tested. Tanhan and Francisco (25) cautioned that the majority of mental health providers tend to utilize reductionist, clinical, individual/intrapersonal approaches that originate from a medical perspective and disregard contextual factors. Mental health professionals including researchers, service providers, and educators could be much more effective and contextually evidence-based when they consider the use of individual and intrapersonal theories within Ecological Systems Theory (EST).

EST is a comprehensive conceptual framework (24) that can be utilized to shape evidence-based studies. Tanhan (23) strongly suggested and explained in detail how mental health professionals can utilize ACT within EST to construct a conceptual framework or a testable theoretical framework. Additionally, it carries great value to involve communities in research and services which requires developing partnership with different community allies based on Community-based Participatory Research (CBPR) to conduct more effective research (24). Such practice recommended by researchers and mental health associations including the American Psychiatric, Counseling, and Psychological Associations. Subsequently, we constructed the proposed conceptual framework based on EST, ACT and CBPR.

\section{Ecological Systems Theory (EST)}

Bronfenbrenner (43) developed EST in the late 1970s to offer a comprehensive developmental framework of multiple factors that provide layers of impact on individuals throughout their lives. Bronfenbrenner described the four main systems that include microsystem, mesosystem, exosystem, and macrosystem which interact to create an individual's dynamic daily contexts (44). The individual is at the center of the model and other systems are nested around them, constantly affecting the individuals as the individual impacts these systems. Given the size and scope of the various ecological systems at play, systems are much more impactful on the individual than the inverse (25). Researchers have reviewed in depth that many researchers from various disciplines have utilized EST to conduct research and to develop and implement effective services (23-25).

\section{Acceptance and Commitment Therapy}

$A C T$, pronounced as one word to remind something meaningful, is placed under the behavioral tradition among the third wave approaches (23,45-47). Tanhan (23) provided a comprehensive metaanalysis of randomized control trials in which the ACT was employed to address diverse biopsychosocial spiritual issues or cultivate wellbeing. ACT has a strong and comprehensive foundation based on functional contextualism, Relational Frame Theory (RFT), and psychological flexibility and inflexibility models (see Table 1 ), which are explained in detail by the researchers $(23,48)$. The 


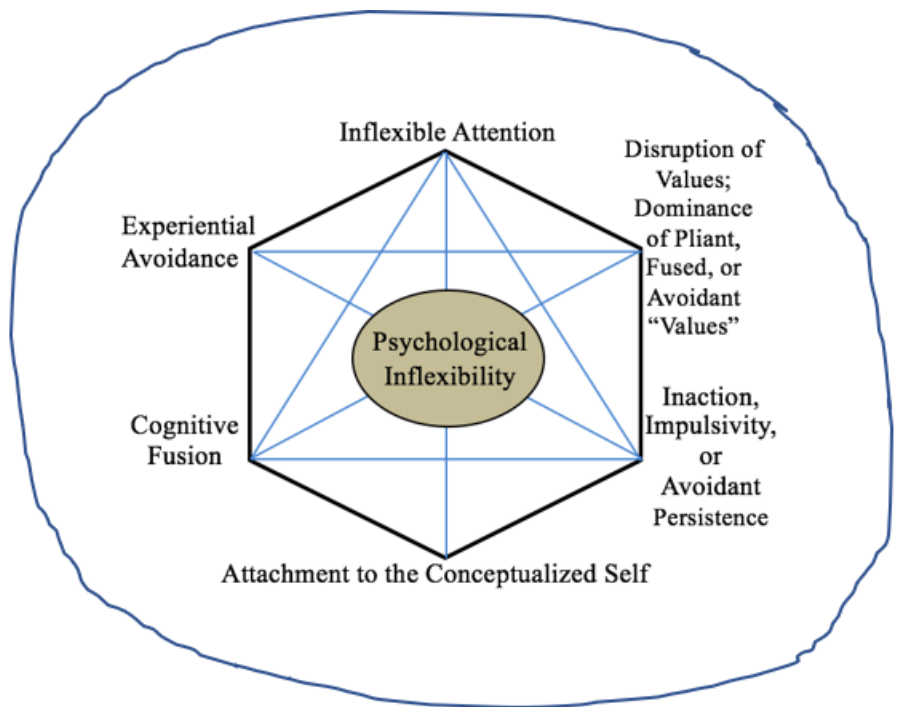

Figure 1. ACT within EST with/for Community Conceptual Framework: ACT Psychological Inflexibility Model from EST perspective: Human Psychopathology

Note. The model explains how human suffering is constructed and gradually becomes psychopathology based on duration, intensity, and lack of professional services. The flexible line encircling the hexaflex represents a contextual perspective as Ecological Systems Theory (EST) suggests considering factors at all levels (e.g., individual, microsystem, macrosystem). The line also represents collaboration with as many as appropriate units (e.g., individuals, families, communities, institutions) at each system simultaneously while trying to understand psychopathology as CBPR researchers suggest. We borrowed the conceptual framework from Tanhan (2019) with permission and used the hexaflex from Hayes and his colleagues (2006).

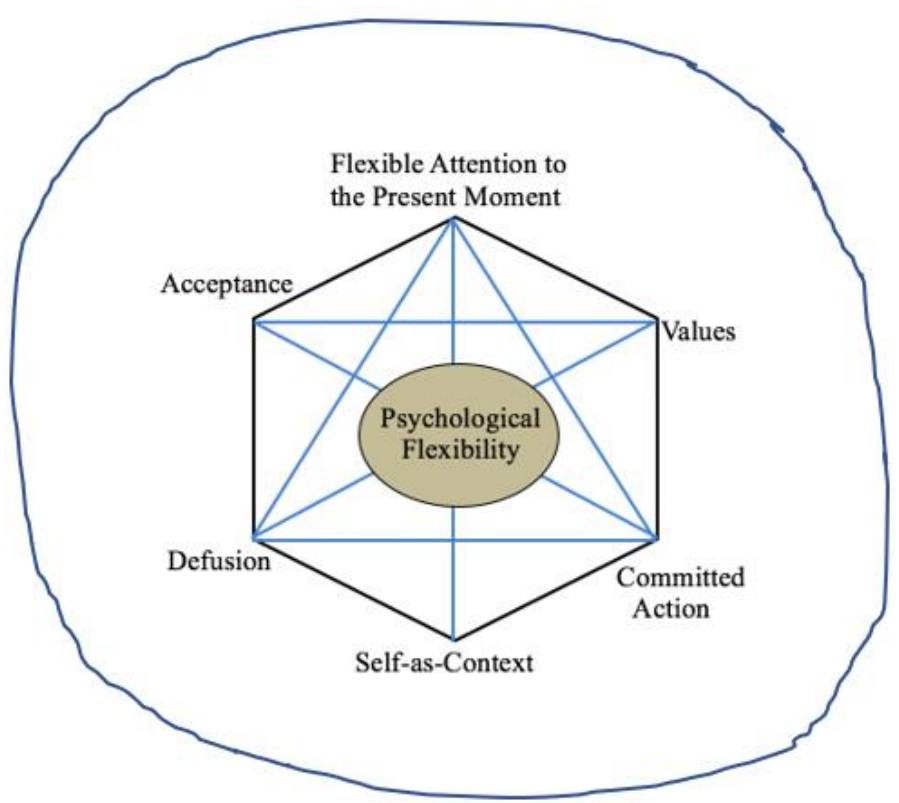

Figure 2. ACT within EST with/for Community Conceptual Framework: ACT Psychological Flexibility Model from EST perspective: Human Wellbeing

Note. The model explains how wellbeing (liveness, a positive psychology) is constructed through psychological flexibility. The flexible line encircling the hexaflex represents a contextual perspective as Ecological Systems Theory (EST) suggests considering factors at all levels (e.g., individual, microsystem, macrosystem). The line also represents collaboration with appropriate partners (e.g., individuals, families, communities, institutions) at each system simultaneously while trying to understand wellbeing as CBPR researcher suggests. We borrowed the conceptual framework from Tanhan (2019) with permission and used the hexaflex from Hayes and his colleagues (2006).

three underlying foundational pieces, and in particular the psychological flexibility model, are brought together in ACT to explain both positive and abnormal psychology $(23,49)$.

The psychological flexibility model is the third and most important component for understanding and properly implementing ACT. The model is based on functional contextualism and RFT. The psychological flexibility model consists of: (a) psychological inflexibility as a model of psychopathology, and (b) psychological flexibility as a model of human functioning, wellbeing, and liveness. The hexagon- shaped psychological inflexibility model comprises six core processes: inflexible attention, disruption of chosen values, inaction or impulsivity, attachment to a conceptualized self, cognitive fusion, and experiential avoidance (see Figure 1). Additionally, the psychologica flexibility model is composed six corresponding core processes: flexible attention to the present moment (present moment awareness), chosen values, committed action, self-as-context, defusion, and acceptance (see Figure 2). 


\section{Community-based Participatory Research (CBPR)}

Collaborating with communities and partners to identify issues or existing resources in order to enhance quality of life is an important component of mental and public health intervention strategy. Though different researchers have used various terminologies such as ally development, Participatory Action Research (PAR; 51), these terminologies serve the same goal of cooperating with community partners to address issues and improve quality of life more effectively (24). CPBR rejects a top down approach to understanding communities and their issues. CBPR is as an alternative research paradigm wherein the researchers collaborate with individuals, groups, communities, and institutions as allies to address issues and enhance wellbeing with and for the community (24,52). Tanhan and Strack (2020) collaborated with a community through an Online Photovoice (OPV) study to explore the community's biopsychosocial, spiritual and economic issues and strengths to address challenges and enhance wellbeing through advocacy. Tanhan and Francisco (25) also used a similar framework to understand and address the issues of a college community and strengthen the already existing support systems. The researchers stressed the importance of codeciding, co-learning, co-working, and activating and building community capacity to reduce disparity among different communities.

Although these six core processes are associated with one another, each of is more deeply interlinked with one or more than with others (49). These inflexibility processes gradually interact with one another leading to biopsychosocial spiritual issues and gradually psychopathology if not addressed professionally and last for a long time (23). Similarly, flexibility processes interact with one another to cultivate wellbeing $(23,48,49)$. In other words, ACT is about providing room for pain, unwanted and unenjoyable feelings, thoughts, sensations and also pleasure or desired feelings, thoughts, and sensations in a mindful and balanced way in the service of facilitating the helping clients to move forward toward chosen values to create a meaningful value-based life and enhance the quality of life and/or address issues $(23,49)$. Mental health professions strive to do this through their service, research, education, and advocacy (50).

\section{ACT within EST with/for Community Conceptual Framework}

Taking into account the extant researchers call for utilizing ACT within EST while considering contextually appropriate collaboration with communities $(23,24)$, in this current study, we proposed the conceptual framework entitled ACT within EST with/for community. Mental health professionals can consider ACT within the EST perspective and collaborate with key community partners to explain how psychopathology and wellbeing is constructed based on the framework for COVID-19 and other contexts. Mental health professionals can use the conceptual framework to develop testable, contextually relevant (i.e., spiritually, culturally, economically) evidence based theoretical frameworks, and robust standardized mental health research and interventions. We added "with/for community" to the conceptual framework from a CBPR perspective to make different and key parts of the community (e.g., general health, mental health, and spiritual institutions and departments; financial institutions; cultural and educational associations; lay people) part of possible research and services: deciding which issues or strengths to be addressed, service provision, evaluation, termination, and follow-up processes. Tanhan and Strack (24) explained in detail how they collaborated with communities using a CBPR approach to explore community issues and then advocate to address these issues and enhance wellbeing through an Online Photovoice (OPV) study. They reported such an approach made the provided services and research much more meaningful and effective at all EST levels, e.g., individual, group, community levels. Such conceptual frameworks are needed in the current and post COVID-19 process to cultivate mental health professionals conducting research and providing services in more contextually effective ways.

\section{Proposed Main Contextual Interventions During COVID-19}

Based on the framework in the context of COVID-19 and Turkey, we suggested the local main mental health services for the city and around to be coordinated from the counseling clinic the first author is leading. The following steps were taken to provide more effective services to all in need for our submitted project. We chose the counseling clinic to be headquarter for coordination. The clinic already had application forms, and we developed all electronic versions of the forms (e.g., intake forms, consent forms, pretests, evaluation forms for each session, and posttests).

We had strong relationships on campus including the department of psychiatry, the department of counseling, formal staff responsible for spiritual activities like imams, student associations, and students or clients who benefited from the clinic. During the COVID-19 process, we announced that the clinics would work to address biopsychosocial, spiritual and economic issues and/or enhance wellbeing. We asked people to share the clinic communication information: phone number, email, and social media accounts. Through these connections we garnered new partners including the chairs of a community mental health center for schizophrenia and bipolar disorders, the organized industrial zone, one of the largest youth community associations, a health association, and about sixty individual volunteers from different professions (e.g., mental health, health, finance, religion, education, lay people) and different levels of the community (e.g., low-middle-high SES, different faith groups) to support the project from their own safe places. Such support was needed to reach out to people in need and make it easy for all to utilize the services in case of stigma around utilizing the services. This leg constructed our CBPR and made it easier to develop the rest of the project. We also got in touch with the nation's largest mental health associations for individual counseling services for referrals, yet their service was too limited from many aspects (e.g., only to some cities, only to people who have COVID-19, only if it is about COVID-19).

Based on the above context, mental health professionals can consider the following interventions, which we proposed in our project. All services will be provided online when needed, by phone.

a. Having a dedicated cell-phone for the project. Having a cellphone dedicated to the project will mobilize the services and also allow people to reach out to the services in face of emergency if they need. The professionals can use the number for social media accounts so that the providers are protected from burnout and possible personal space and time violations. 
b. Provide online training to all who will be in the project team and be part of the process about and the flow of the program. We planned to receive some help from a professional counseling company that has many mental health professionals with expertise in ACT and EST. They allocated a mental health team for helping with training all involved in the project, supporting the project team in terms of mental health services if needed, being part of supervising the process throughout the process, and being ready for compensating the team in case of lack of shortage for providing the services mentioned below.

c. Online or phone-based psychiatric interview. The leading mental health associations and insurance company across the world (e.g., American Psychiatric, Psychology, and Counseling Associations) found that providing online mental health services is as effective as providing in person services. However, there is lack of research support for these ideas in many countries, and we are not aware of any research both in public or private institutions about online or phone based psychiatric interviews in Turkey.

d. Online individual, couple, and group counseling: Providing online or phone based counseling is a highly needed service in this process because unprecedented COVID-19, its results, and intensive and long-term physical and social isolation can be very detrimental in terms of biopsychosocial spiritual and economic aspects. For these services an important number of mental health professionals with counseling experience are need and if possible seasoned and with a PhD degree may be more effective. Therefore, for these services we had approximately 25 mental health professionals who at least had a master's degree and five years of experience providing counseling. Volunteers would provide two individual or two group counseling sessions per week due to project requirement of not allocating money to service providers and to prevent the volunteers from burn-out. The project description had strict rules on how to allocate funds and more effort is needed to facilitate the authorities understand that mental health providers need to be paid for providing counseling. It is ineffective for mental health providers to provide counseling volunteer for free for a long time, which has been the case in Turkey during COVID-19. This led to some serious unprofessional situations; for example, a client reported that they found a flyer on social media and got in touch for free counseling, yet the counselor abruptly stopped providing counseling after the first session in which the client shared their story. Such an experience might cause a negative attitude and intention toward approaching and utilizing the services for this client or the general public. Another client shared they reached out to the nation's largest mental health associations' phone that was provided for mental health services and yet the client was not provided with services. The association told the client that their severe anxiety was not related to COVID-19.

e. Online Read-Reflect-Share (ORRS) group bibliotherapy: The first author of this paper constructed the ORRS and it has been the most popular mental health service at the counseling clinic for the last three years. The participants favored Online RRS more than face-to-face RRS because in online RRS the participants have much more flexibility in terms of time, materials, number of groups, and having one or two group facilitators who are trained peer counselor-in-education. In our submitted project, online RRS group bibliotherapy is the largest service with plans to have 2,000 participants in 100 groups (each consisting of 10 to 20 participants) facilitated by trained counselors-in-training and professional mental health providers. We have used the following books in online RRS: Reality Slap and Happiness Trap by Russ Harris. Slow Down by Kemal Sayar was used in an earlier face-to-face RRS group. In order to have a contextually more effective online RRS for the project (a) we consulted with allies and asked them to review the content and recommend other books; (b) improved available ORRS protocols (i.e., explaining RRS, introducing mindfulness, time, sessions) to make them more appropriate for COVID-19 context, (c) allocated one third of our project budget on buying physical books and delivering them to participants because many clients who joined online RRS strongly recommended materials in print; we were not able to provide the physical book to few participants and they had to read from the scanned materials. Further, in the current COVID-19 context, people spend too much time on technology; therefore, in the project, (d) we offered participants the option of attending individual or group counseling sessions after completing eight sessions of the ORRS group, and (e) we also provided participants with various group options (e.g., only men-women-adolescents, only spiritual leaders-teachers-health workers-college students) to be contextually sensitive. For example, the researchers (14) in China developed psychological interventions for frontline health providers during COVID-19 process and yet the health providers did not utilize the services at all due to a lack of time. However, in our ORRS, we had a few frontline health providers during the COVID-19 outbreak and they attended all online RRS sessions and reported them to be very meaningful. They also referred other health providers who waitlisted for ORRS, we currently have about 200 who are willing to be in the next online RRS groups.

\section{DISCUSSION}

Our findings suggest that there is lack of well-structured and grounded mental health services and research in the context of COVID-19 in Turkey. Though there were a small number of individual services (some with serious malpractice issues), there were few mental health services provided by national mental health associations which were focused on individual counseling online or by phone and only when the issues were directly related to COVID-19. This seem to be very problematic because human biopsychosocial spiritual economic issues are related to one another and also past, present, and future. Additionally, to our knowledge, none of these were based on a well-grounded project or a comprehensive framework. We also did not find any peerreviewed manuscripts regarding online mental health services and research on COVID-19 response within Turkey and similarly no studies on online bibliotherapy. Researchers recommended 
utilizing ACT as a framework to address wellness and psychopathology for general yet especially with college students $(22,23,53)$. Additionally, many researchers found online mental health or telemental health to be effective during the pandemic experience (28-33), which support our preliminary results, personal experience from the ground during this pandemic, and our proposed framework. Though we provided the book for free to most of the participants, it is important to pay attention to social justice because we were not able to support some of the participants who were willing to participate and yet were not able to afford internet or an electronic device (e.g., phone, computer) to stay connected with online RRS group member and the clinic. We asked them to waitlist and benefit from the project once it is approved.

Based on all these, as the COVID-19 pandemic continues to affect Turkey and the rest of the world, our findings and ongoing experience from the counseling clinic and online counseling, RRS, and collaboration with key community members, and the proposed conceptual framework provided vital guidance for creating contextual and evidence-based well-grounded mental health interventions and research to address the issues and enhance wellbeing.

\section{LIMITATIONS}

There are several limitations to the proposed approach. First and foremost, online mental health can put the already marginalized at greater risk of not being able to gain access to mental health services and create further mental health disparities. This creates a serious social justice issue for individual with low SES, which is a crucial issue in Turkey. Second, online mental health services are not well studied compared to as face-to-face services. Third, relying on volunteers put such services' long-term sustainability at risk. With the exception of ORRS, we had limited experience with online or phone based psychiatric interviews and individual, couple, and group counseling. Therefore, the following implications should be considered in light of these limitations.

\section{IMPLICATIONS}

The key implications of this study could be classified into four specific areas of mental health: practice, research, training, and advocacy.

\section{Mental Health Providers}

The primary implication of this study is the use of ACT from EST perspective while collaborating with community partners from a CBPR perspective to create a contextual, biopsychosocial, and spiritually sensitive online mental health services. The providers can maximize the services with limited capacity to serve clients during COVID-19 pandemic providing support at individual, family, group, community, institutional, and national levels to enhance wellness and address psychopathology.

A specific implication is to use ACT within EST and collaborate with key community partners $(23,54)$ to reach thousands of clients through ORRS as explained above. Many practitioners fail to attend to the influence of larger system factors (e.g., national and international politics, global issues) and creating space to examine how these affect clients, in particular underprivileged communities across the systems (e.g., microsystem, mesosystem, exosystem, macrosystem). COVID-19 affects all and yet mostly individuals and communities with less privileges, therefore, it is crucial to be more specific about how to provide services in such cases. The providers can be much more effective by individualizing services for various population groups to shorten gaps rather than expanding it among groups. For example, mental health providers can be socially more justice paying attention to participants' SES. First, the providers should strive mindfully to make sure they have reached out to people with low, middle, and high SES, while striving to include at least about \%50 percent of the participants from low and \%30 from the middle SES to cultivate a balanced mental health stability rather than causing more disadvantageous at the community level. Providing services fairly throughout the systems will provide the real and unique resilience both at individual and community levels (Tanhan, 2019; Tanhan \& Francisco, 2019). For example, the providers can work on providing basic electronic devices (telephone, computer) or at least find ways to provide free internet or at least other free resources (free credits) for phone-calls especially for people with low SES so that they can benefit from the services. Another important, economically and contextually doable implication is providing free mental health (especially in online bibliotherapy services) materials especially to people with less privileges. This is important because in many countries (e.g., Turkey) empirically well-grounded mental health books are expensive and unavailable (e.g., not being familiar with, local sores not selling such books) for many communities in their primary contexts (e.g., neighborhood, local stores).

The next implication, the providers can be more effective by tailoring the content and flow of the services (e.g., reading materials, books, apps, videos) considering the participants' biopsychosocial spiritual and economic contexts (23). For instance, the participants who have or prefer less synchronized online resources can have more offline services (e.g., individual bibliotherapy, chatting/typing, self-reflective writing, telemental health) with the providers to stay connected with psychologically well-grounded services. Additionally, the providers can strive to tailor the content of the services based on the perspectives (e.g., unique cultural factors, values, spirituality, religiosity) most matter to the participants especially when serving marginalized and disprivileged groups to be able to attend them from a more holistic perspective. In such an approach, the participants will feel more heard, felt, human, flourished and connected to larger society and humanity rather becoming more isolated and discriminated and overloaded with biopsychosocial spiritual and economic issues. Another important implication could be, especially for online group bibliotherapy or group counseling is to provide group options (e.g., only men, women, LGBTQ+; only lowmiddle-high SES; mild-moderate-severe psychopathology level; only health providers, mental health providers, spiritual/religious leaders, teachers; only college students; only 15-16-17 years old teenagers) to enhance participation and effect of the services. Last and foremost, the mental health professionals need to pay utmost attention to people with special needs (e.g., having blindness, deafness, physical handicaps, the speaking language as a second or third language in where they live due to being immigrant or refugee) to provide services accordingly because these people are more likely to face more issues and benefit less from mental health services. The providers should make sure they reach out to 
these people with special needs and who have low SES because in such a case the disadvantages and issues are layered and are multiplied that can lead much more serious issues for all in the long run.

In sum, mental health providers are encouraged to play their crucial role, while protecting themselves as well, in this difficult COVID-19 process to cultivate a more conscious, mindful, and theory-based perspective to ground their practice and research. In such a conscious approach, the providers will be able to promote and contribute to social justice for all without causing even more disparities during this difficult pandemic process.

\section{Mental Health Researchers}

The primary implication for research is the importance of constructing a theoretical framework based on the conceptual framework to conduct online empirical studies (e.g., case, quasi-experimental, randomized control studies, OPV) and psychoeducation for individual, family, group, health and mental health professionals, spiritual leaders, and community levels to address biopsychosocial and spiritual issues and enhance wellbeing. Tanhan (23) called for the use of ACT with EST to conduct online services and research. As the main coordinator of the clinic, the first author received more than 60 surveys from mental health researchers whom asked him to send the surveys to the clients at the clinic. Almost all the researchers did not have an institutional review board permissions and the researchers did not provide a clear framework for their research when the first author asked for such information. Additionally, most of the research as the authors we received were surveys and quantitative, and some researchers discuss whether such research manipulate the participants and prevent understanding their unique perspective $(24,25)$. Therefore, based on the researchers' suggestions and considering COVID-19 process, future researchers can use OPV to understand factors that cause issues or enhance wellbeing during the pandemic. In Turkey, institutional review boards are not well regulated and during COVID-19 most of them did not run smoothly. All these indicate that improvement is need at individual researchers and institutional levels to conduct robust research. Therefore, researchers and research institutions like universities can benefit from the proposed conceptual framework to become more organized during this COVID-19 and afterward to conduct well-grounded robust and standardized research with big data.

\section{Mental Health Educators}

Mental health educators are the most critical key individuals for enhancing the quality of mental health services (50). The most important implication in this domain would be introducing EST, ACT, and CBPR while paying utmost attention to stress how to utilize these online for research and service especially in the midst of pandemics. A more specific point is to introduce the proposed conceptual framework and interventions to mental health providers in-training so that they improve their competency level to act from a more contextual perspective and collaborate with the ones in need of services. The educators can support this introduction with more testable theoretical frameworks and practical readings, visitors from different communities involved, and role-plays so that the framework and training become much more contextualized and practical (23).

\section{Mental Health Advocacy}

Mental health professionals could utilize the above mentioned practice, research, training implications from the ACT within EST with/for the community framework perspective to inform policy makers and authorities. In this way, the professionals can advocate flexibly and simultaneously to enhance wellbeing at various social levels during the COVID-19 in a more effective way through activating available system's and community's resources. Informing and involving the key policy maker is very crucial because it is very common to see key policy makers and authorities dismiss the importance of mental health (24). Furthermore, the providers can do little without the key ones' support (25). Therefore, we strived to include representatives from a range of institutions, departments, organization, and associations as partners based on CBPR and EST. Additionally, mental health professionals can utilize the proposed conceptual framework to reach out to authorities and provide a system that allow for conducting robust, standardized, well-grounded mental health research through collecting large data sets through one or multiple centers. The ministry of health regularly shares other large data related to physical health symptoms and their treatment (e.g., filiation process, number of tests, intubation, cases) related to COVID-19. However, they have not, to date, shared results or basic statistics about mental health.

\section{CONCLUSION}

Most people in Turkey have had difficult and unenjoyable experiences (mild to severe levels) based on various factors (e.g., province, SES, and history of mental health) during the initial phase of the COVID-19 pandemic $(8,11,18)$. These first natural responses gradually became more biopsychosocial, spiritual and economic issues with the gradual and extended precautions being taken and having insufficient mental health services. For example, we received disproportionate demand for mental health services with the most being for ORRS at the clinic where the first author leads mental health services and research. Based on the belated call for mental health project, we submitted the content of this manuscript to the government to extend already existing mental health services to serve the larger public.

Preliminary and quasi experimental mental health research at the clinic showed that receiving individual face-toface or online mental health services (individual counseling, RRS group bibliotherapy) was associated with lower anxiety and depression levels and higher quality of life and psychological flexibility (34). Therefore, mental health professions can utilize this framework to formulate more specific and contextually appropriate psychological interventions, giving more attention to online services. The use of such evidence based and contextually appropriate services while collaborating with as many different partners as possible from different levels of EST and paying utmost attention to include vulnerable and underprivileged populations will increase the effectiveness of services while enhancing social justice and wellbeing in a more balanced way across the levels of the society. 


\section{REFERENCES}

1. World Health Organization. Naming the coronavirus disease (COVID-19) and the virus that causes it. Published online 2020, April 20. Available at: https://www.who.int/ emergencies/diseases/novel-coronavirus-2019/technicalguidance/naming-the-coronavirus-disease-(covid-2019)and-the-virus-that-causes-it

2. Huang C, Wang Y, Li X, Ren L, et al. Clinical features of patients infected with 2019 novel coronavirus in Wuhan, China. The Lancet, 2020;395(10223):497-506. https://doi. org/10.1016/S0140-6736(20)30183-5 PMID: 31986264

3. Bao Y, Sun Y, Meng S, Shi J, Lu L. 2019-nCoV epidemic: Address mental health care to empower society. The Lancet, 2020;395(10224):e37-e38. https://doi.org/10.1016/ S0140-6736(20)30309-3 PMID:32043982

4. Mills K. (Host). (2020-present). Bonus episode: Coronavirus Anxiety [Audio podcast]. Speaking of psychology. Available at: https://traffic.libsyn.com/secure/sopapa/Bonus_Epi sode_-_Coronavirus_Anxiety_with_Baruch_Fischhoff_PhD _mixdown.mp3

5. Özdin S, Bayrak Özdin Ş. Levels and predictors of anxiety, depression and health anxiety during COVID-19 pandemic in Turkish society: The importance of gender. International Journal of Social Psychiatry. Published online May 8, 2020. https://doi.org/10.1177/0020764020927051 PMID:32380879

6. Taylor S, Landry C, Paluszek M, Fergus TA, McKay D, Asmundson GJ. Development and initial validation of the COVID stress scales. Journal of Anxiety Disorders. 2020;72:102232. https://doi.org/10.1016/j.janxdis.2020. 102232 PMID:32408047

7. Wang C, Pan R, Wan X, Tan Y, et al. Immediate psychological responses and associated factors during the initial stage of the 2019 coronavirus disease (COVID-19) epidemic among the general population in China. International Journal of Environmental Research and Public Health, 2020;17(5):1729. https://doi.org/10.3390/ijerph17051729 PMID:32155789

8. Yildirim M, Arslan G. Exploring the associations between resilience, dispositional hope, subjective well-being, and psychological health among adults during early stage of COVID-19. 2020. https://doi.org/10.31234/osf.io/vpu5q

9. Ahorsu DK, Lin CY, Imani V, Saffari M, Griffiths MD, Pakpour $\mathrm{AH}$. The fear of COVID-19 scale: Development and initial validation. International Journal of Mental Health and Addiction. 2020. https://doi.org/10.1007/s11469-02000270-8 PMID:32226353

10. Haktanir A, Seki T, Dilmaç B. Adaptation and evaluation of Turkish version of the fear of COVID-19 scale. Death Studies. Advance Online Publication. 2020. https://doi.org/10.1080/07481187.2020.1773026

11. Satici B, Gocet-Tekin E, Deniz ME, et al. Adaptation of the fear of COVID-19 scale: Its association with psychological distress and life satisfaction in Turkey. International Journal of Mental Health Addiction. Published online May 8, 2020. https://doi.org/10.1007/s11469-020-00294-0 PMID:32395095

12. Zandifar A, Badrfam R. Iranian mental health during the COVID-19 epidemic. Asian Journal of Psychiatry, 2020;51:101990. https://doi.org/10.1016/j.ajp.2020.101990 PMID:32163908
13. Cortese S, Asherson P, Sonuga-Barke E, Banaschewski T, et al. ADHD management during the COVID-19 pandemic: Guidance from the European ADHD Guidelines Group. The Lancet Child \& Adolescent Health. 2020;4:412-4. https://doi.org/10.1016/S2352-4642(20)30110-3 PMID:32311314

14. Chen Q, Liang M, Li Y, Guo J, et al. Mental health care for medical staff in China during the COVID-19 outbreak. The Lancet Psychiatry, 2020;7(4):e15-e16. https://doi.org/ 10.1016/S2215-0366(20)30078-X PMID:32085839

15. Mamun MA, Griffiths MD. First COVID-19 suicide case in Bangladesh due to fear of COVID-19 and xenophobia: possible suicide prevention strategies. Asian Journal of Psychiatry, 2020;51:102073. https://doi.org/10.1016/ j.ajp.2020.102073 PMCID: PMC7139250

16. Aljofan M, Gaipov A. COVID-19 treatment: The race against time. Electronic Journal of General Medicine, 2020;17(6):em227. https://doi.org/10.29333/ejgm/7890

17. Arslan G, Yıldırım M., Tanhan A, Bulus M, Allen, KA. Coronavirus stress, optimism-pessimism, psychological inflexibility, and psychological health: Psychometric properties of the Coronavirus Stress Measure. International Journal of Mental Health and Addiction. 2020. https://doi.org/10.1007/s11469-020-00337-6

18. Çiçek I, Tanhan A, Bulus M, Arslan G. Psychological inflexibility predicts depression and anxiety during COVID19: Acceptance and commitment therapy perspective. Manuscript submitted for publication. 2020.

19. Liu S, Yang L, Zhang C, Xiang YT, et al. Online mental health services in China during the COVID-19 outbreak. The Lancet Psychiatry, 2020;7(4):e17-e18. https://doi.org/10.1016/ S2215-0366(20)30077-8 PMID: 32085841

20. Yao H, Chen JH, Xu YF. Rethinking online mental health services in China during the COVID-19 epidemic. Asian Journal of Psychiatry, 2020;50:102015. https://doi.org/10.1016/j.ajp.2020.102015 PMID:32247261

21. Ministry of Health in Turkey (2020). Türkiye'deki güncel durum. Available at: https://covid19.saglik.gov.tr/

22. McDonnell S, Semkovska M. Resilience as mediator between extraversion, neuroticism, and depressive symptoms in university students. Journal of Positive Psychology and Wellbeing, 2020;4(1):21-40. Available at: http://journalppw.com/index.php/JPPW/article/view/164

23. Tanhan A. Acceptance and commitment therapy with ecological systems theory: Addressing Muslim mental health issues and wellbeing. Journal of Positive Psychology and Wellbeing, 2019;3(2):197-219. Available at: http://journalppw.com/index.php/JPPW/article/view/172

24. Tanhan A, Strack RW. Online photovoice to explore and advocate for Muslim biopsychosocial spiritual wellbeing and issues: Ecological systems theory and ally development. Current Psychology, 2020. Published online March 30, 2020. https://doi.org/10.1007/s12144-020-006926

25. Tanhan A, Francisco VT. Muslims and mental health concerns: A social ecological model perspective. Journal of Community Psychology, 2019;47(4):964-78. https://doi.org /10.1002/jcop.22166 PMID:30730559

26. Savci M, Griffiths MD. The development of the Turkish craving for internet gaming scale (CIGS): A validation study. International Journal of Mental Health and Addiction. Published online August 2, 2019. https://doi.org/10.1007/ s11469-019-00114-0 
27. Duan L, Zhu G. Psychological interventions for people affected by the COVID-19 epidemic. The Lancet Psychiatry, 2020;7(4):300-2. https://doi.org/10.1016/S2215-0366(20) 30073-0 PMID:32085840

28. Golberstein E, Wen H, Miller BF. Coronavirus disease 2019 (COVID-19) and mental health for children and adolescents. JAMA Pediatrics. Published online April 14, 2020. https://doi.org/10.1001/jamapediatrics.2020.1456 PMID:32286618

29. Knopf A. Addiction telemedicine comes into its own with COVID-19. Alcoholism \& Drug Abuse Weekly, 2020;32(13):5 6. https://doi.org/10.1002/adaw.32673

30. Ohannessian R, Duong TA, Odone A. Global telemedicine implementation and integration within health systems to fight the COVID-19 pandemic: A call to action. JMIR Public Health and Surveillance, 2020;6(2):e18810. https://doi.org/10.2196/18810 PMID:32238336

31. Whaibeh E, Mahmoud $\mathrm{H}, \mathrm{Naal} \mathrm{H}$. Telemental health in the context of a pandemic: The COVID-19 experience. Current Treatment Options in Psychiatry. Published online April 2, 2020. https://doi.org/10.1007/s40501-020-00210-2 PMID:32292687

32. Wright JH, Caudill R. Remote treatment delivery in response to the COVID-19 pandemic. Psychotherapy and Psychosomatics, 2020;89(3):1. https://doi.org/10.1159/ 000507376 PMID:32213775

33. Zhou X, Snoswell CL, Harding LE, Bambling M, et al. The role of telehealth in reducing the mental health burden from COVID-19. Telemedicine and e-Health, 2020;26(4):377-9. https://doi.org/10.1089/tmj.2020.0068 PMID:32202977

34. Tanhan A, Arslan G, Uğur E, Uzun E, et al. A contextually sensitive bibliotherapy based on acceptance and commitment therapy and ecological systems theory perspectives. In Acceptance and Commitment Therapy \& Relational Frame Theory (ACT \& RFT) Congress. 2019, 6-9 November. Istanbul, Turkey. 2019.

35. Temel MK, Ertin H. Grip pandemisi kıssasından COVID-19 pandemisine hisseler. Anadolu Kliniği Tıp Bilimleri Dergisi, 1918;25(Supplement 1):63-78. https://doi.org/10.21673/ anadoluklin.716868

36. Dost B, Koksal E, Terzi Ö, Bilgin S, et al. Attitudes of anaesthesiology specialists and residents toward patients infected with the novel coronavirus (COVID-19): A national survey study. Surgical Infections. 2020:350-6. https://doi.org/10.1089/sur.2020.097 PMID:32275463

37. Halaçlı B, Kaya A, Topeli A. Critically-ill COVID-19 patient. Turkish Journal of Medical Sciences, 2020;50:585-91. https://doi.org/10.3906/sag-2004-122 PMID:32293831

38. Hasöksüz M, Kiliç S, Saraç F. Coronaviruses and SARS-CoV2. Turkish Journal of Medical Sciences, 2020;50:549-56. https://doi.org/10.3906/sag-2004-127 PMID:32293832

39. Petersen E, Gökengin D. SARS-CoV-2 epidemiology and control, different scenarios for Turkey. Turkish Journal of Medical Sciences, 2020;50:509-14. https://doi.org/10.3906/ sag-2003-260 PMID:32283894

40. Açikgöz Ö, Günay A. The early impact of the Covid-19 pandemic on the global and Turkish economy. Turkish journal of medical sciences, 2020;50(SI-1):520-6. https://doi.org/10.3906/sag-2004-6 PMID:32283904
41. Bostan S, Erdem R, Öztürk YE, Kılıç T, Yılmaz A. The effect of COVID-19 pandemic on the Turkish society. Electronic Journal of General Medicine, 2020;17(6):em237. https://doi.org/10.29333/ejgm/7944

42. Tanhan A, Young JS. Muslims and mental health services: A concept map and a theoretical framework. Manuscript submitted for publication. 2020.

43. Bronfenbrenner U. Toward an experimental ecology of human development. American Psychologist, 1977;32(7):513-31. https://doi.org/10.1037/0003-066X.32.7. 513

44. Bronfenbrenner U. The ecology of human development. Harvard university press. 1979.

45. Hayes SC. Acceptance and commitment therapy, relational frame theory, and the third wave of behavioral and cognitive therapies. Behavior Therapy, 2004;35(4):639-65. https://doi.org/10.1016/S0005-7894(04)80013-3

46. Hayes SC. ACT immersion: An introduction to ACT as a process-based therapy [Online ACT course]. 2019. Available at: https://act.courses

47. Yavuz F, Ulusoy S, Iskin M, Esen FB, et al. Turkish version of Acceptance and Action Questionnaire-II (AAQ-II): A reliability and validity analysis in clinical and non-clinical samples. Klinik Psikofarmakoloji Bülteni-Bulletin of Clinical Psychopharmacology, 2016;26(4):397-408. https://doi.org/10.5455/bcp.20160223124107

48. Hayes SC, Luoma JB, Bond FW, Masuda A, Lillis J. Acceptance and commitment therapy: Model, processes and outcomes. Behaviour Research and Therapy, 2006;44(1):1-25. https://doi.org/10.1016/j.brat.2005.06.006

49. Hayes SC, Strosahl KD, Wilson KG. Acceptance and commitment therapy: The process and practice of mindful change (2nd ed.). New York, NY: The Guilford Press. 2012.

50. Tanhan A. Beginning counselors' supervision in counseling and challenges and supports they experience: Based on developmental models. Adiyaman Üniversitesi Eğitim Bilimleri Dergisi, 2018;8(1):49-71. https://doi.org/10.17984/ adyuebd.336222

51. Minkler M. Using participatory action research to build healthy communities. Public Health Reports, 2000;115(23):191. https://doi.org/10.1093/phr/115.2.191 PMID: 10968753

52. Wallerstein NB, Duran B. Using community-based participatory research to address health disparities. Health Promotion Practice, 2006;7(3):312-23. https://doi.org/ 10.1177/1524839906289376 PMID:16760238

53. Soares D, Woods K. An international systematic literature review of test anxiety interventions 2011-2018. Pastoral Care in Education, 2020. Published online February 18, 2020. https://doi.org/10.1080/02643944.2020.1725909

54. Kaplaner K. Spirituality in the context of acceptance and commitment therapy (ACT). Spiritual Psychology and Counseling, 2019;4(3):351-8. https://doi.org/10.12738/ spc.2019.4.3.067 